\title{
Serum and Urine Uromodulin Levels in Patients Who Had Computed Tomography with lodized Contrast Agent
}

\author{
Erdem Çankaya ( $\nabla$ dr25erdem@gmail.com ) \\ Atatürk University Medical Faculty \\ Adem Karaman \\ Atatürk University Medical Faculty \\ Muhammet Çelik \\ Atatürk University Medical Faculty \\ Hasan DOĞAN \\ Atatürk University Medical Faculty \\ Abdullah Uyanık \\ Atatürk University Medical Faculty \\ Can Sevinç \\ Atatürk University Medical Faculty \\ Bülent Albayrak \\ Atatürk University Medical Faculty \\ Muhammet Akif Güler \\ Atatürk University Medical Faculty \\ Murat Altunok \\ Atatürk University Medical Faculty
}

\section{Research Article}

Keywords: Uromodulin, contrast agent, biomarker, urine levels, serum level

Posted Date: February 16th, 2022

DOI: https://doi.org/10.21203/rs.3.rs-1249647/v1

License: (c) (1) This work is licensed under a Creative Commons Attribution 4.0 International License. Read Full License 


\section{Abstract}

\section{Background}

Uromodulin is a protein produced in kidney tubule cells and found in the urine. Uromodulin levels detected in serum and urine provide information about renal tubule functions and reserve.

\section{Methods}

In this study, in individuals with normal kidney functions; the short-term changes in uromodulin levels in both urine and serum were evaluated before and after contrast agent administration 86 patients were included in the study. Serum and urine uromodulin levels were measured before and 24 hours after contrast agent administration.

\section{Results}

Although there was no significant change in glomerulifiltration rate before and after contrast agent administration $(98.7 \pm 17.5$, $100.8 \pm 18.5, p=0.1$, respectively), serum uromodulin values decreased significantly $(43.4 \pm 17.6,24.8 \pm 17.9, p \leq 0.05$, respectively). Urinary uromodulin levels increased significantly $(670 \pm 175,805 \pm 340, p \leq 0.05$, respectively).

\section{Conclusions}

Serum uromodulin level can be a biomarker that can be followed in ischemic kidney injury or after administration of nephrotoxic agents. Urinary uromodulin levels can provide information about renal susceptibility to nephrotoxic agents.

\section{Introduction}

Uromodulin (Tamm-Horsfall protein), the most abundant protein in urine under physiological conditions, was discovered by Tamm and Horsfall in the 1950s. It is a mucoprotein produced by epithelial cells in the thick ascending limb of the loop of Henle of the nephron (1). Its serum and urine levels enable it to be used as a biomarker for tubular mass and kidney functions (2). In addition, in the studies carried out; It has been shown that it plays an important role in maintaining water and electrolyte balance, regulating albumin excretion, provides protection against immunomodulation and thus urinary tract infections, prevents kidney stones, and may be a predictive biomarker for cardiovascular events (3-8).

Contrast agents (CA) are now widely used to improve the Computed Tomography (CT) scan image and in the diagnosis of trauma, neoplastic disease, and inflammatory diseases. Complications such as contrast-induced nephropathy (CIN) can be seen as a result of this widespread use. Studies on the incidence of Acute Kidney Injury (AKI) after CA have reported that it varies in a wide frequency range, between $2 \%$ and $25 \%$ in some cases, depending on the clinical situation and type (9-11). The pathophysiology of CIN is not fully understood. It is thought to occur with the triggering of many mechanisms together. Intrarenal vosoconstriction, direct tubular damage and oxidative stress were considered to be the main factors $(12,13)$. To date, no definitive treatment has been found for AKI developing following radiocontrast administration. However, prevention still remains the cornerstone of CIN. Therefore, a careful analysis of risk factors is required and new studies are being conducted on CIN predictability.

Although serum creatinine ( $\mathrm{SCr}$ ) level is used in the diagnosis of AKI, it is not an ideal biomarker because it increases late and depends on many factors such as age, gender, and muscle mass. Kidney damage that does not impair glomerular filtration rate (GFR) does not cause a change in serum creatinine and may not affect urine output. This level of damage was termed 'subclinical' because it was below the clinical threshold for detection. Molecules such as kidney injury molecule 1 (KIM-1), neutrophil gelatinase-associated lipocalin (NGAL), metalloproteinase inhibitor 2 (TIMP2), insulin-like growth factor binding protein 7 (GFBP7) have been used to determine this damage (14).

Our aim in this study is to evaluate the change in blood and urine levels of uromodulin before and after CA administration in individuals with normal kidney function.

\section{Material And Method}


The study was an observational study $(n=86)$ involving men and women aged 17 years and over. Participants had no known diagnosis of kidney disease. All participants gave informed consent and the local institutional ethics committee approved the study methods( Ataturk University Faculty of Medicine Clinical Research Ethical Box. No: B.30.2 AT;0.01.00/12). Before the procedure, both blood and urine samples were taken from the patients who were admitted to the radiology clinic and whose contrast-enhanced CT would be taken (a bolus of $80 \mathrm{ml}$ of nonionic contrast material $(350 \mathrm{mg}$ iyot $/ \mathrm{ml}$ ) was administered using a dual syringe automatic injector connected to the cannula placed in the antecubital vein at of low rote of $4 \mathrm{ml} / \mathrm{s}$ followed by a $20 \mathrm{ml}$ saline flush at $2.5 \mathrm{ml} / \mathrm{s}$ ) from various outpatient clinics. After 24 hours of intravenous administration of contrast material, the same patient group was called to the nephrology outpatient clinic and blood and urine samples were taken again.

\section{Exposure}

Blood samples were taken into gel biochemistry tubes with $5 \mathrm{cc}$ and kept at room temperature for 20 minutes to coagulate. After coagulation, the serum samples obtained after centrifugation at $3500 \mathrm{rpm}$ for 15 minutes were aliquoted. After the urine samples were taken into the urine tubes, they were centrifuged at 2000rpm for 20 minutes, and the supernatant parts were taken and aliquoted. Aliquots were stored at $-80^{\circ} \mathrm{C}$ until the working day. The samples were studied according to the Uromodulin ELISA kit instructions on the study day (code E-EL-H0941, brand ELABSCIENCE, UROMODULIN HUMAN ELISA KIT).

\section{Statistical analysis}

SPSS 20.0 (SPSS Inc., Chicago, IL, USA) program was used for data analysis. In the statistical analysis of the study, mean, standard deviation, frequency and percentage values were defined. The general characteristics and demographic characteristics of the groups were determined by Frequency (Descriptive analysis: frequency analysis for a single variable) analysis. In pairwise comparisons; The Independent Sample t-Test was used to compare the mean of two independent groups, and the Paired Sample t-Test was used to compare a single variable for 2 different conditions. Chi-square test was used to determine the relationship between categorical variables. Receiver Operating Characteristic (ROC) analysis; It was used to determine the sensitivity and specificity of age in the increase of Uromodulin level in the urine and to predict the cut-off value. A $p \leq 0.05$ value was considered statistically significant in the entire study.

\section{Results}

52 of the participants were female and 34 were male. Their mean age was $51.02 \pm 13.8$ years. 27 of the volunteers had hypertension $(H T)$, diabetes mellitus (DM), or both. Glomerofiltration rates (eGFR) of the volunteers were calculated with the Chronic Kidney Disease Epidemiology Collaboration (CKD-EPI) formula before and after intravenous (IV) contrast administration. Their values were $98.7 \pm 17.5$ $\mathrm{ml} / \mathrm{min}$ and $100.8 \pm 18.5 \mathrm{ml} / \mathrm{min}$, respectively, and no significant difference was detected $(p=0.1)$. Other demographic data of the participants are given in Table 1. No significant increase in SCr value was detected in any of the participants. The serum uromodulin levels (SUMOD) measured were significantly decreased compared to the baseline level in $97.7 \%$ of the participants after contrast administration (fig. 1,Table 1).

Urinary uromodulin levels (UUMOD) were found to be significantly higher in urine samples collected simultaneously (Table 1). UUMOD levels were not homogeneous like SUMOD levels (Figure 2). 52 of the participants (60.4\%) had an increased level of UUMOD (Table 2). Two significant differences were found in our analyzes between the subgroups with decreasing and increasing UUMOD levels (Table 2). First, the group with decreasing UUMOD had significantly higher GFR than the group with increasing UUMOD. Second, the mean age of the group with decreasing UUMOD was significantly lower (Table 2). According to our ROC analysis; The cut-off value was found to be 49.5 years using the Youden index. Age can be used as a parameter for the increase or decrease of urinary uromodulin level (Figure 3$)(p=0.009)$. When the urinary uromodulin levels of the groups with decreased and increased UUMOD levels were compared before and after contrast administration, it was shown that the decrease and increase were significant (Table 3).

\section{Discussion}

Uromodulin is a mucoprotein produced in the thick ascending limb of the loop of henle and less commonly in the distal convoluted tubule of the kidney. The produced Uromodulin undergoes proteolytic cleavage by all serine proteases in the apical membrane and is released in the urine to form high molecular weight polymers. By unknown mechanisms, smaller amounts of uromodulin are also transported to its basolateral part to be released into the interstitium and circulation. Some of the uromodulin is sent to the basolateral 
thick ascending limb membrane, and blood uromodulin is consistently detected at lower levels than in urine, suggesting basolateral release of uromodulin with regional vasculature and release into the systemic circulation (15). CA are directly toxic to tubular epithelial cells, leading to loss of function and both apoptosis and necrosis. In addition, renal vasoconstriction that occurs with impaired vasodilation is an important mechanism that causes kidney damage (16). In our study, the reason for the decrease in SUMOD levels after contrast agent administration may be the decrease in blood flow due to vasoconstriction in the vessels providing renal blood supply, and therefore the decrease in uromodulin released into the circulation from the basolateral segment of tubule cells. The increase in UUMOD confirms that the decrease in SUMOD is not due to tubular uromodulin production. This suggests that SUMOD levels may be a biomarker in ischemic damage to the kidney. Serum uromodulin level is positively correlated with eGFR in healthy individuals. It was not detected in stage 5 CKD. It detects potential functional kidney resources and starts to increase in plasma after renal transplantation. Beside this, it was reported that higher serum uromodulin concentration is correlated with proper metabolic profile and also independently from eGFR and other cardiovascular risk factors it is related with lower mortality risk $(7,8,17)$. Recently, circulating uromodulin has been shown to be an important regulator of systemic oxidative stress through inhibition of TRPM2 channels. There are studies reporting the relationship between SUMOD and inflammation (18).

Caroline Liu et al. found that although SUMOD levels before and after coronary angiography were decreased, there was no significant difference between patients who did not develop CIN and those who did (19). The differences were that the patient groups had a higher mean age, lower eGFR ( $48 \pm 13 \mathrm{ml} / \mathrm{min})$, SUMOD levels were checked sooner after contrast was applied, and the amount of contrast was not mentioned compared to the groups in our study. In our study, there was no patient who developed CIN. However, the use of terminology for subclinical kidney injury, where creatinine does not increase and GFR does not decrease, is currently being discussed (14). It suggests the idea that kidney health represents a tubular tissue marker independent of glomerular filtration markers and could potentially help detect early kidney damage (7). It has been shown that UUMOD level is a biomarker of functional kidney reserve and is associated with innate immunity $(2,20)$. Puthumana $J$ et al. confirm the role of UUMOD as an important protective factor in the maintenance of kidney function and that UUMOD may be an indicator of adaptive tubular repair (20). That is, for repair to occur, damage must occur. The increase in UUMOD after contrast in our study suggests that it may be a result of this repair process. The fact that the eGFR of the group with increased UUMOD was lower than the group with decreasing UUMOD supports this. It is known that the sensitivity to nephrotoxic agents is increased in people with reduced renal functional reserve. However, the high mean age of the group with increased UUMOD level in our study suggests that it is due to the decrease in renal reserve as a natural process with age.

As a result, SUMOD decreases rapidly independent of eGFR after exposure to contrast agent. It may be a biomarker followed in ischemic kidney injury or after exposure to nephrotoxic agents. While UUMOD is variable after contrast agent exposure, age and eGFR are important parameters in this variability. UUMOD can provide information about renal susceptibility to nephrotoxic agents.

\section{Study limitations}

Our study has strengths and limitations. The effect of possible additional factors has been reduced because it was performed in individuals with normal kidney functions, not in a specific patient group, the amount of contrast agent was standardized and the same group was compared. However, the small size of our patient group and the lack of long-term follow-up are the limitations of our study. Nevertheless, our study is one of the rare studies that provide information about the sudden change in serum and urine uromodulin levels.

\section{Declarations}

\section{Ethics declarations.}

All participants gave informed consent and the local institutional ethics committee approved the study methods( Ataturk University Faculty of Medicine Clinical Research Ethical Box. No: B.30.2 AT;0.01.00/12)" 'Declarations Section'- 'Ethics approval and consent to participate' sub-section.

We declare that all methods are carried out in accordance with the relevant guidelines and regulations.

We declare that written consent has been obtained from all participants.

\section{Consent for publication}


Not applicable.

\section{Competing interests}

The authors declare that they have no conflict of interest

\section{Availability of data and materials}

Datasets used and/or analyzed during the current study are publicly available on

(https://urldefense.com/v3/_https://docs.google.com/document/d/1_MtRv8RIYCqTK2tvbmw2UunkuL_IFAbz69vwks4c9U/edit? usp=sharing_;!!NLFGqXoFfo8MMQ!8K8XQq2eB5PBJDf-TfVb8z79_CUob_yxGOcfb5t6-y7zceAHPUGmI38dqGqAML_xWyCCat3u\%24) and

(https://urldefense.com/v3/_https://docs.google.com/spreadsheets/d/1hDzPFMB753XI4Y04UaTYIQAN5rkMGt5F0rkdLpPI7Ns/edit? usp=sharing_;!!NLFGqXoFfo8MMQ!8K8XQq2eB5PBJDf-TfVb8z79_CUob_yxGOcfb5t6-

y7zceAHPUGmI38dqGqAML_xW9aqDUYS\%24) and are available from the corresponding author upon reasonable request".

\section{Funding}

This work was supported by the [Atatürk universty BAP] under Grant [TAP-9346 number].

\section{Authors' contributions}

EÇ, AK: Conceptualization, Methodology, Software

EÇ, MÇ: Data curation, Writing- Original draft preparation.

HD, M.AG: Visualization, Investigation..

AU, BA: Supervision

C S, M A: Software, Validation.

EÇ, MA, AK: Writing- Reviewing and Editing

\section{Acknowledgements}

Not applicable.

\section{References}

1. Tamm, I. \& Horsfall, F. L. Jr. Characterization and separation of an inhibitor of viral hemagglutination present in urine. Proc. Soc. Exp. Biol. Med. 1950;74, 106-108.

2. Pruijm M, Ponte B, Ackermann D. et all. Associations of Urinary Uromodulin with Clinical Characteristics and Markers of Tubular Function in the General Population. Clin J Am Soc Nephrol. 2016; 11 (1): 70-80

3. Ole Torffvit, Olle Melander, U Lennart Hultén. urinary excretion rate of Tamm-Horsfall protein is related to salt intake in humans. Nephron Physiol actions. 2004;97(1):p31-6.

4. C Thorup, A Ollerstam, A E Persson, O Torffvit. Increased tubuloglomerular feedback reactivity is associated with increased NO production in the streptozotocin-diabetic rat. J Diabetes Complications. 2000;14(1):46-52.

5. Pennica, D. et al. Identification of human uromodulin as the Tamm-Horsfall urinary glycoprotein. Science.236, 83-88 (1987).

6. Leiherer A, Muendlein A, Saely CH. et all. Serum uromodulin is a predictive biomarker for cardiovascular events and overall mortality in coronary patients. Int J Cardiol. 2017.15;231:6-12. 
7. Graciela E Delgado, Marcus E Klebe, Scharnagl H.et all. Serum Uromodulin and Mortality Risk in Patients Undergoing Coronary Angiography. J Am Soc Nephrol. 2017;28(7):2201-2210.

8. Devuyst O, Olinger E, Rampoldi L. Uromodulin: from physiology to rare and complex kidney disorders. Nat Rev Nephrol. 2017;13(9):525-544.

9. Goto Y, Koyama K, Katayama S, et al. Influence of contrast media on renal function and outcomes in patients with sepsisassociated acute kidney injury: a propensity-matched cohort study. Crit Care. 2019;23(1):249.

10. Cely CM, Schein RM, Quartin AA. Risk of contrast-induced nephropathy in the critically ill: a prospective, case-matched study. Crit Care. 2012;16(2): 67

11. Rachoin J-S, Wolfe Y, Patel S.et all. Contrast associated nephropathy after intravenous administration: what is the magnitude of the problem? Renal Failure.2021; 43:1, 1311-1321.

12. McCullough PA, Soman SS. Contrast-induced nephropathy. Crit Care Clin 2005;21:261-80.

13. Tumlin J, Stacul F, Adam A, et all. Contrast-induced nephropathy: Clinical insights and practical guidance - A report from the CIN consensus working panel. Am J Cardiol 2006; 986 Suppl 1:1-78.

14. Kellum JA, C Ronco, R Bellomo. Conceptual advances and evolving terminology in acute kidney disease. Nature Reviews. 2021:

$17,493-502$

15. Kipp A, Olinger E. What Does Uromodulin Do? CJASN.2021; 16: 150-153.

16. Mehran R, Dangas GD, Weisbord SD. Contrast-Associated Acute Kidney Injury. N Engl J Med 2019;380:2146-55.

17. ScherberichJE, GruberR, NockherWA, ChristensenEl, SchmittH, Herbst V, Block M, Kaden J, SchlumbergerW: Serum uromodulin-A marker of kidney function and renal parenchymal integrity.Nephrol Dial Transplant. 2018;33: 284-295.

18. LaFavers KA, Macedo E, Garimella PS. et all. Circulating Uromodulin inhibits systemic oxidative stress by inactivating the TRPM2 channel. Sci Transl Med. 2019;02; 11(512):

19. Liu C, Mor MK,. Palevsky, PM et all. Postangiography Increases in Serum Creatinine and Biomarkers of Injury and Repair. CJASN15:2020:1240-1250.

20. Puthumana J, Philbrook HT, Xu L. Et all. Biomarkers of inflammation and repair in kidney disease progression. J Clin Invest. 2021;131(3):e139927

\section{Tables}

Table 1: Demographic characteristics of patients, values before and after contrast agent 


\begin{tabular}{|llll|}
\hline $\mathrm{n}=86$ & Values & & \\
\hline Gender (Female, $\mathrm{n})$ & 51 & & \\
\hline Age (years) & $51.02 \pm 13.8$ & \\
\hline BMI (kg/m²) & $29 \pm 4.6$ & & \\
\hline HT (n) & 15 & & \\
\hline DM $(n)$ & 6 & & \\
\hline HT and DM (n) & 6 & & \\
\hline & Before CA & After CA & p \\
\hline SUMOD (ng/ml) & $43.4 \pm 17.6$ & $24.8 \pm 17.9$ & $\leq 0.01$ \\
\hline UUMOD (ng/ml) & $670 \pm 175$ & $805 \pm 340$ & $\leq 0.01$ \\
\hline eGFR (ml/min) & $98.7 \pm 17.5$ & $100.8 \pm 18.5$ & 0.1 \\
\hline Creatinine (mg/dl) & $0.75 \pm 0.19$ & $0.72 \pm 0.18$ & 0.034 \\
\hline
\end{tabular}

HT: Hypertension, DM: Diabetes mellitus, BMI: Body mass index, CA: Contrast agent, SUMOD: Serum uromodulin levels, UUMOD: Urinary uromodulin levels, eGFR: Estimated glomerular filtration rate

$p<.01$ value was considered statistically significant

Table 2: Evaluation of patients according to urinary uromodulin levels

\begin{tabular}{|llll|}
\hline & UUMOD Decreasing Group $(\mathrm{n}=\mathbf{3 4})$ & UUMOD Increasing Group $(\mathrm{n}=\mathbf{5 2})$ & $\mathrm{p}$ \\
\hline Gender & & & \\
\hline Female $(\mathrm{n}=51)$ & 23 & 28 & 0.2 \\
\hline Male $(\mathrm{n}=\mathbf{3 5})$ & 11 & 24 & \\
\hline Age (years) & $46.5 \pm 13.2$ & $53.9 \pm 13.6$ & $\leq 0.01$ \\
\hline BMI (kg/m $\left.{ }^{2}\right)$ & $28.5 \pm 4.8$ & $29.6 \pm 4.5$ & 0.7 \\
\hline eGFR Before CA (ml/min) & 104 & 94 & $\leq 0.01$ \\
\hline eGFR After CA (ml/min) & 107 & 96 & $\leq 0.01$ \\
\hline SUMOD Before CA (ng/ml) & 41.6 & 44.6 & 0.4 \\
\hline SUMOD After CA $(\mathrm{ng} / \mathrm{ml})$ & 21.3 & 27.1 & 0.14 \\
\hline HT and DM & 9 & 18 & \\
\hline
\end{tabular}

BMl: Body mass index, CA: Contrast agent, SUMOD: Serum uromodulin levels, UUMOD: Urinary uromodulin levels, eGFR: Estimated glomerular filtration rate, HT: Hypertension, DM: Diabetes mellitus,

$p<.01$ value was considered statistically significant

Table 3: Comparison of pre- and post-contrast urinary uromodulin levels of UUMOD decreasing and increasing groups 


\begin{tabular}{|llll|}
\hline & UUMOD Before Contrast Agent & UUMOD After Contrast Agent & $p$ \\
\hline $\begin{array}{l}\text { UUMOD Decreasing } \\
\text { Group ( } \mathrm{n}=\mathbf{3 4} \text { ) }\end{array}$ & $698 \pm 226$ & $511 \pm 162$ & $\leq 0.01$ \\
\hline $\begin{array}{l}\text { UUMOD Increasing } \\
\text { Group }(\mathrm{n}=\mathbf{5 2})\end{array}$ & $652 \pm 130$ & $997 \pm 283$ & $\leq 0.01$ \\
\hline
\end{tabular}

UUMOD: Urinary uromodulin levels,

$p<.01$ value was considered statistically significant

\section{Figures}

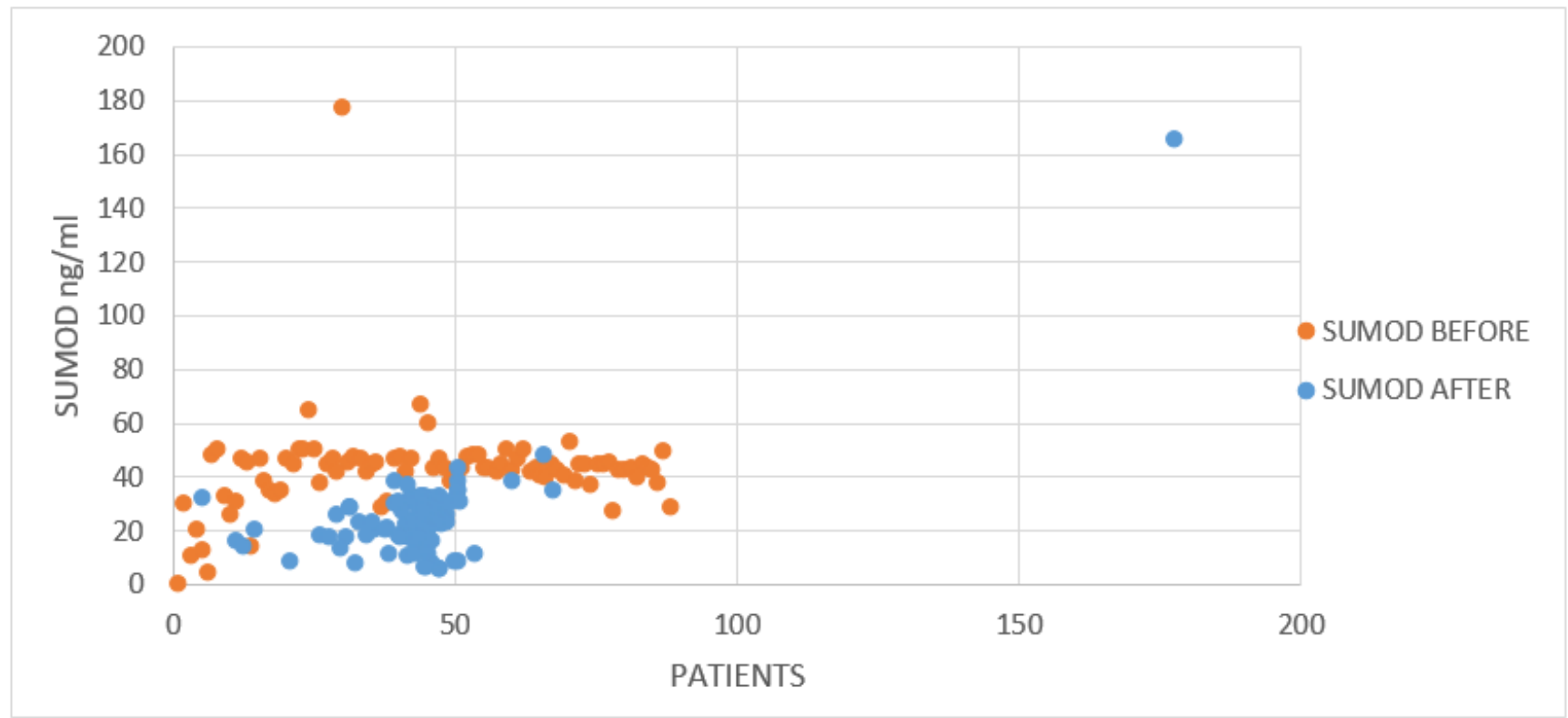

\section{Figure 1}

Distribution of serum uromodulin levels before and after contrast agent

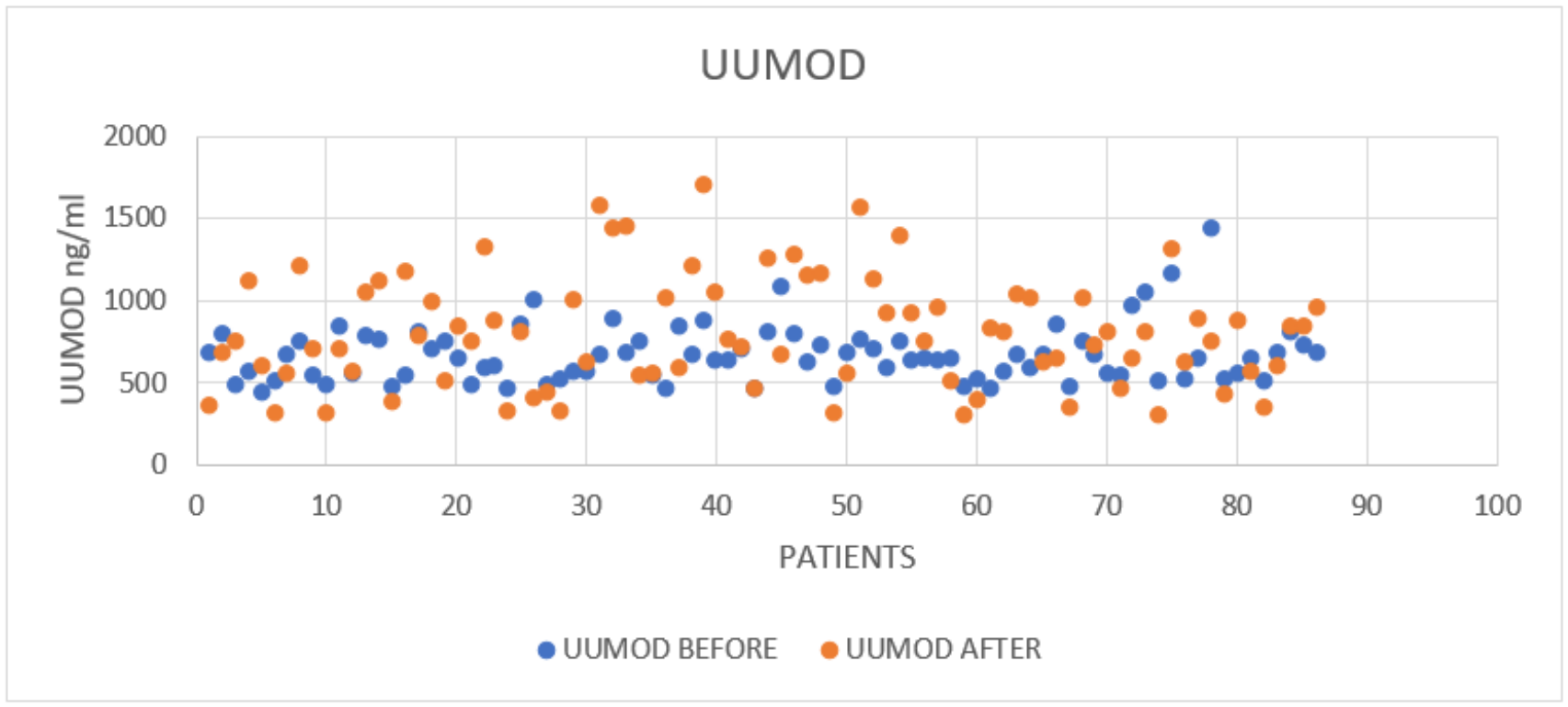

Page $8 / 9$ 


\section{Figure 2}

Distribution of urinary uromodulin levels before and after contrast agent

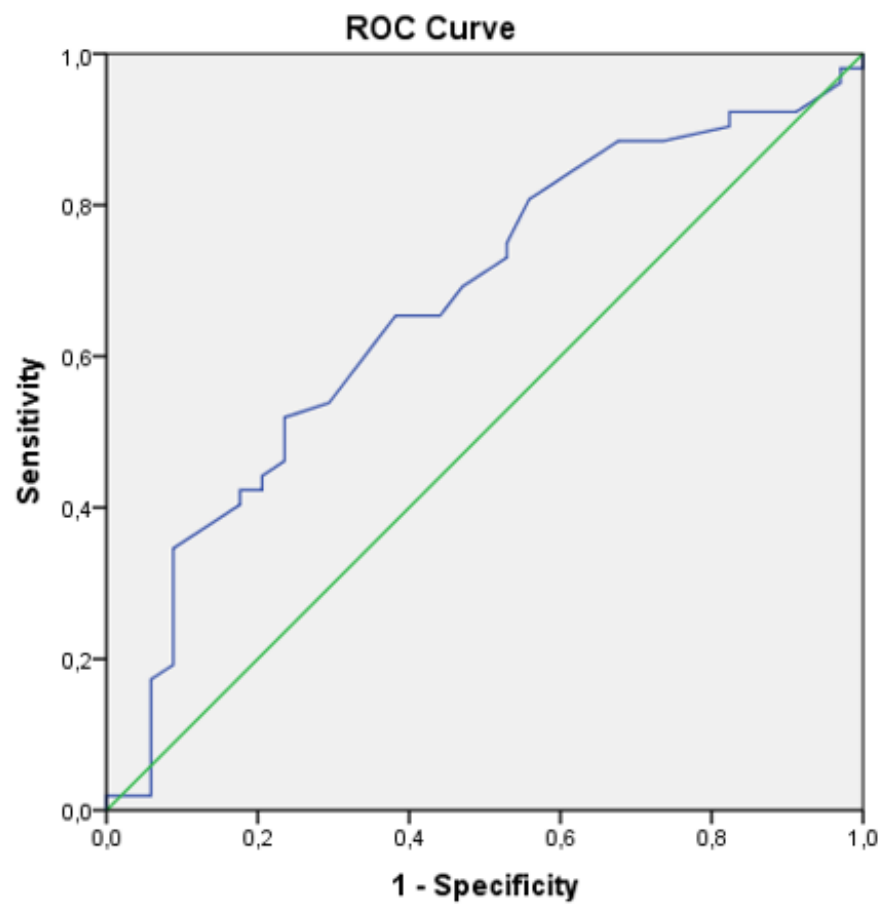

Diagonal segments are produced by ties.

\section{Figure 3}

According to ROC Analysis; The cut-off value was found to be 49.5 years using the Youden index. Age can be used as a parameter for the increase or decrease of urinary uromodulin level $(p=0.009)$. 